\title{
Chapitre VII - Les équipements
}

\section{Maurice Wolkowitsch}

\section{(2) OpenEdition}

\section{Journals}

Édition électronique

URL : https://journals.openedition.org/rhcf/1320

DOI : 10.4000/rhcf.1320

Éditeur

Rails \& histoire

Édition imprimée

Date de publication : 10 février 2004

Pagination : 224-248

ISBN : 0996-9403

ISSN : 0996-9403

Référence électronique

Maurice Wolkowitsch, "Chapitre VII - Les équipements », Revue d'histoire des chemins de fer [En ligne], 30 | 2004, mis en ligne le 22 août 2012, consulté le 22 avril 2022. URL : http://journals.openedition.org/ rhcf/1320; DOI : https://doi.org/10.4000/rhcf.1320 


\section{Chapitre VII}

\section{Les équipements}

工 a construction d'une voie ferrée suppose des travaux nécessite l'acquisition de matériel ; une interdépendance étroite lie ces trois termes : le matériel doit être adapté à la voie qu'il empruntera et réciproquement, comme au trafic auquel il devra répondre. Les bâtiments doivent être conçus en prévision du trafic espéré et de son potentiel de croissance; la voie doit pouvoir acheminer tous les mouvements nécessaires pour faire face à la demande.

\section{La voie}

Les distances parcourues généralement courtes, les trafics modestes ne poussaient pas à réaliser des progrès analogues à ceux obtenus par les grands réseaux. La recherche de la vitesse intervient tardivement avec l'utilisation des autorails et non par des progrès notables intervenus la traction à vapeur; la vitesse est incompatible avec les déclivités et les rayons des courbes adoptés lors de la construction.

Les rayons des courbes s'abaissent à $80 \mathrm{~m}$ sur les lignes à voie normale, ils se tiennent le plus souvent entre 150 et $350 \mathrm{~m}$ et atteignent exceptionnellement $500 \mathrm{~m}$. Ils sont sur les lignes à voie métrique dans des cas constatés dans 41 départements en dessous des $50 \mathrm{~m}: 20 \mathrm{~m}$ dans les Pyrénées-Orientales et dans le Bas-Rhin, $25 \mathrm{~m}$ en Gironde, $30 \mathrm{~m}$ dans sept départements ; ces situations se rencontraient à l'approche des villes et très souvent sur des tracés sur route. Dans treize départements, on relève des déclivités dépassant ou atteignant $20 \%$; sur voie normale, dans certains cas, on ne s'y attendrait pas : Landes, Nord, Somme... Pour la voie métrique, compte tenu des lignes qui sont liées à la route, on ne peut être surpris de l'importance des déclivités : si on s'en tient aux départements où cet écartement est présent, on trouve des déclivités égales ou inférieures à $30 \%$ dans $38 \%$ d'entre eux, comprises entre 31 et $41 \%$ dans $35 \%$, entre 41 et $50 \%$ dans $11 \%$ : l'Isère, les départements pyrénéens et la Haute-Vienne offrent les taux les plus élevés (voir les travaux de R. Guyot, annexe 1).

1- (Note de la page précédente) Les données relatives aux matériels et aux personnels employés sur les réseaux des TV et de TVB ont été regroupées dans le chapitre VI consacré à ces entreprises. Des équipements moins diversifiés que pour les CFIL et les TVM, une simplification dans la composition hiérarchique des personnels dans la réalité, sinon dans le cadre institutionnel ont conduit à ce choix. 
Faible trafic et vitesse réduite orientent les constructeurs vers l'emploi de rails d'un poids permettant un tonnage à l'essieu inférieur à $10 \mathrm{t}$; le rail vignole est choisi pour son coût inférieur de $20 \%$ au rail à double champignon. Cet armement de la voie avec un poids au mètre de 18 à $20 \mathrm{~kg}$ pour l'écartement métrique concerne $95 \%$ du réseau ; le poids s'abaisse à $15 \mathrm{~kg}$ sur six réseaux et atteint $30 \mathrm{~kg}$ sur le chemin de fer de La Mure destiné à assurer un trafic houiller. La voie de 1,44 m a un même type de rail, mais le poids métrique est plus élevé, compris entre 25 et $30 \mathrm{~kg}$.

Dernière caractéristique évoquée, l'implantation de la voie en site propre (52\% du réseau) ou sur chaussée et accotement des routes $(48 \%$ ). Dans ce dernier cas, outre l'inconvénient d'une plate-forme partagée avec d'autres usagers, figurent les paramètres de tracé et de profil qui sont ceux de la route, corrigés par de très rares travaux d'art : déclivités et rayons des courbes sont moins favorables. Huit départements ont toutes leurs lignes en site propre, onze toutes leurs lignes sur route. On trouve dans les deux cas des départements très peuplés ou non, des départements de plaines et de montagnes, des réseaux de quelques kilomètres et de plus de 300 kilomètres. Les décisions prises résultaient d'une volonté politique où intervenait le volume des investissements publics à consentir et, aussi, du choix des options techniques des services administratifs qui avaient à approuver le projet et des avis des ingénieurs qui le réaliseraient.

Le service des Voie et Bâtiments était démuni en encadrement dans les petites compagnies. L'entretien de la voie était confié à une main-d'œuvre peu formée, dont une partie de manœuvres recrutés à la journée ; cette situation engendrait de nombreuses défaillances dans les services et causait des accidents souvent sans gravité. Les problèmes de maintenance n'étaient pas moindres pour les matériels.

\section{Les bâtiments}

\section{. Les ateliers}

Le patrimoine ferroviaire offre de multiples exemples de ces installations : dépôts et ateliers. L'une a fait face pendant un siècle (19022001) à l'entretien des matériels du B.A. à Romorantin : les cinq autorails Verney de 1951 et les deux autorails CFD Socofer de 1983 sortis des ateliers de Bagnères-de-Bigorre y étaient encore entretenus en 2000. L'arrivée en 2001 d'autorails d'une nouvelle génération, l'XTR 74500, a exigé pour sa maintenance de nouvelles installations plus performantes, conçues suivant une architecture devant s'inscrire dans le site. Souhaitons 
que les anciens ateliers, qui sont encore en état et qui témoignent d'une remarquable transmission des savoir-faire et d'une continuité dans leurs équipements et outillage seront mis au service de la préservation des matériels historiques aujourd'hui en service sur les réseaux touristiques.

Les ateliers de la SGCFE à Cosne-d'Allier n'ont pas connu une semblable longévité. Mis en service en 1892, pour entretenir le matériel du réseau du Centre de la compagnie avec un hangar de $1000 \mathrm{~m}^{2}$, deux agrandissements en 1893 et 1906 les ont dotés de près de $2000 \mathrm{~m}^{2}$ et d'un bâtiment surélevé abritant un pont transbordeur; encore en 1936, un atelier était créé pour l'entretien des autorails. Toute activité a cessé en 1952. La commune avait contribué à l'acquisition des terrains, le département et l'État à égalité étaient intervenus pour financer la construction. Au début du siècle, les ateliers employaient 150 ouvriers $^{2}$, encadrés par un ingénieur, un inspecteur de la Traction, un chef de dépôt et d'atelier, un chef-magasinier. Seule une très grande entreprise pouvait disposer d'une main-d'œuvre avec des représentants de tous les échelons de la hiérarchie ${ }^{3}$.

\section{. La multiplication et la diversité des points d'arrêt}

Sur les grands réseaux, la construction des bâtiments a obéi progressivement à des normes exprimées en $\mathrm{m}^{2}$ en fonction des trafics prévisibles : bureau, salles d'enregistrement des bagages, de distribution des billets, d'attente, quais, cours et halles à marchandises, espaces réservés au transbordement entre compagnies, partagés parfois avec des compagnies secondaires, tout était défini pour faire face aux besoins, de la halte à la gare terminus avec rebroussement sans omettre la hiérarchie intermédiaire des stations et des gares. Cela explique la similitude des aspects et de l'organisation des gares, malgré certaines concessions faites à l'architecture régionale 4 .

Sur les réseaux secondaires, une exploitation simplifiée, les prévisions de trafics modestes, la recherche d'économies ont conduit à multiplier les points d'arrêt facultatif, sans personnel, dotés éventuellement pour tout équipement d'un quai herbeux. Les haltes et stations ouvertes au seul trafic des voyageurs ont un préposé, un bâtiment de faible surface, parfois une chambre en étage ; souvent le préposé habite au village et sa présence se limite aux heures de passage des trains;

\footnotetext{
2- Les corps de métiers les plus divers étaient présents : ajusteurs, tourneurs, chaudronniers, ferreurs-wagon, forgerons, menuisiers, peintres, serruriers, charrons, tapissiers.

3- R. Courtaud, «Un exemple de valorisation de la France centrale par le rail : «Le Tacot» à Cosne-d'Allier 1887-1950», RHCF 24-25, p. 342-358.

4- P. Blanc, Agenda Dunod, « Chemins de fer», p. 69-78.
} 
l'installation peut être une simple baraque en planches ; le réseau d'Illeet-Vilaine en porte témoignage à la station de Pont-Béan. Les stations ouvertes aussi au trafic des marchandises disposent d'espaces semblables à celui des stations équivalentes des grands réseaux ; photographies et, mieux, patrimoine en survie (Argentat, Treignac, Vatan) ${ }^{5}$ en fournissent d'innombrables exemples. Les gares peu nombreuses s'inspirent dans leurs aspects, leur plan, leur organisation des conceptions retenues sur les grands réseaux. Quelques grandes compagnies ont construit une ou deux gares de plus grandes dimensions avec un effort sur le plan de l'esthétique : Sud-France à Nice, les Chemins de fer vicinaux à Vesoul et Gray illustrent ce propos ${ }^{6}$.

Une particularité des chemins de fer secondaires est la faible distance entre les points d'arrêt, comprise en moyenne entre 1,5 et $2 \mathrm{~km}$, grâce à la multiplication des arrêts facultatifs, ces deniers sont plus nombreux sur les lignes tracées sur route; dans le cas fréquent où les chemins de fer secondaires ont un parcours urbain, l'espacement entre points d'arrêt y est particulièrement faible. La volonté d'une desserte de proximité est manifeste.

\section{. La conservation du patrimoine bâti}

Toute circulation a disparu sur la majorité du réseau depuis un demi-siècle, voire davantage. Un patrimoine important nous a été légué, il est menacé ; beaucoup d'associations se soucient de son avenir avec raison ; ne serait-il pas nécessaire de faire le départ entre le possible et l'illusoire pour rendre leur action plus efficace? Deux objectifs peuvent être poursuivis. Le premier vise à trouver une utilisation sociale à l'emploi d'un bâtiment dont l'entretien sera assuré par l'administration, les collectivités territoriales ou par les associations qui en ont l'usage : la poste centrale de Vesoul, la gendarmerie de Graçay, la halte de Reboursin devenue chapelle dans cette commune sans église, des salles des fêtes communales, autant d'exemples de ce qui peut être réalisé ; à l'heure où de petites communes programment à grands frais la construction d'une salle des fêtes ou l'extension de leurs bureaux, il y a bien souvent des opportunités à saisir, si le bâtiment n'est pas excentré7.

5- J. Banaudo, Trains oubliés, vol. 3, PO - Midi, Menton, Éd. du Cabri, 1982, 152 p. « Les gares d'Argentat et Treignac », p. 59-60, sont sur une ligne concédée au PO avec construction à voie métrique ; l'exploitation a été assurée à l'origine par une compagnie secondaire : la Société d'exploitation des chemins de fer de Corrèze.

6- J. Finsterwald et C. Bouchaud, «Les chemins de fer vicinaux de la Haute-Saône de l'extension (1878-1917) au déclin (1918-1938) », Chemins de fer régionaux et urbains, $\mathrm{n}^{\circ} 287\left(2001, \mathrm{n}^{\circ} 5\right)$ p. $4-15$.

7- B. Moreau, Le Tacot, petit train berrichon, 2 tomes, Châteauroux, 1988, 122 p. et Issoudun, $1989,176 \mathrm{p}$. 
L'appropriation privée peut s'accompagner ou non d'un souci de conservation, mais seul l'aspect extérieur est visible pour le public ${ }^{8}$. Un second objectif peut être cherché : la protection au titre de monument historique ; cela peut concerner très peu de bâtiments, éventuellement des ouvrages d'art. Les dossiers doivent être circonstanciés: originalité de l'architecture, technique et matériaux de construction, âge, inscription dans le site, esthétique, intérêt pour le public, possibilité de visite et d'animation; la liste n'est pas exhaustive?. Sachant que les dossiers doivent obtenir une première approbation régionale, la chance du moins ne pourrait venir que d'un regroupement des associations d'une région qui uniraient leurs efforts en soutenant trois ou quatre projets qu'elles auraient sélectionnés ; cela n'empêcherait pas chaque association de se battre sur le plan local. Les deux objectifs sont compatibles.

L'ingénieur est un personnage essentiel dans la réalisation d'une ligne de chemin de fer ; ce n'est pas un hasard si on en trouve souvent à l'origine des projets. Leur compétence s'exerce dans le choix des matériels, des rails, des traverses, des plaques tournantes, mais aussi dans celui du tracé offrant le meilleur profil en long tout en répondant à la demande. La correction des inégalités du relief devait être obtenue sans multiplier les travaux d'art onéreux ; l'idéal était de suivre la route et d'éviter le passage en site propre. Cette politique d'économies avait ses limites ; elle imposait la multiplication de fortes rampes et de courbes à faibles rayons, mais il fallait tout de même une exploitation rentable avec des trains d'un tonnage suffisant et roulant à une vitesse acceptable.

La recherche poussée d'économies dans la construction des chemins de fer secondaires menait à une politique qu'il y aurait avantage à comparer avec les dépenses engagées à la même époque dans la réalisation des lignes du plan Freycinet. Plus généralement, la comparaison entre toutes les solutions techniques retenues sur les réseaux secondaires et sur les lignes d'intérêt général, compte tenu des obligations qui leur étaient respectivement imposées, serait riche d'enseignement, et éclairerait l'avenir respectif et les conditions de l'exploitation des unes et des autres.

8- Des cheminots ont acheté à leur départ en retraite une station dont ils ont conservé en l'état le rez-de-chaussée (salles d'attente, de distribution des billets, bureau et tout le matériel et de belles affiches très anciennes) ; ils s'inquiètent pour l'avenir. Il s'agit de la station de Mers-sur-Indre que j'ai pu visiter par hasard. Elle est sur une ligne d'intérêt général, mais cela ne doit pas être un cas unique et pourrait servir d'exemple.

9- Voir Jean Fosseyeux, "Conditions et effets de la protection des immeubles et meubles au titre de la loi de 1913 sur les monuments historiques et consistance du patrimoine ferroviaire protégé : quelques exemples ", Revue d'histoire des chemins de fer, 28-29 (2003), p. 462-475. 


\section{Les matériels}

L'attention sera portée ici au matériel roulant comprenant les engins de traction, les voitures, fourgons et wagons, chaque type divisé, si nécessaire, en catégories. Les très nombreuses études consacrées à des lignes ou à des entreprises présentent des analyses minutieuses des matériels en service; nous en signalons deux exemples, d'autres pourraient être proposés. Nous nous attacherons ici à dégager des vues plus globales. Les données relatives à la progression du parc visent la période de 1901 à 1911.

\section{. Allongement du réseau et croissance du parc de matériel}

Le rapide allongement des réseaux au début du siècle s'accompagne du développement du parc. Le kilométrage exploité offre un taux de croissance supérieur à celui du parc de matériel ; l'amélioration des méthodes d'exploitation permet une meilleure rotation du matériel, déjà favorisée par le seul renforcement du réseau. Le parc pour le transport des marchandises sur les TVM connait les plus grandes progressions (tabl. 40).

Tableau 40. Taux de croissance comparés du kilométrage exploité et des matériels utilisés entre 1901 et 1911 (\%)

\begin{tabular}{|l|c|c|c|c|}
\hline & Kilom étrage & Locom otives & Voitures & Wagons \\
\hline CFIL & 101 & 74 & 86 & 85 \\
\hline TVM & 103 & 90 & 72 & 97 \\
\hline
\end{tabular}

L'affectation du matériel à chaque ligne ou réseau traduit la volonté de chiffrer l'investissement en matériel occasionné par l'ouverture de nouvelles liaisons et d'intégrer ce coût à l'ensemble des dépenses d'établissement ; le rassemblement de ces données était indispensable à ceux qui assumaient la responsabilité de décider d'entreprendre ou non la construction d'une nouvelle voie ferrée. Dans la pratique, les matériels d'une même entreprise ont été utilisés sur l'ensemble des parties contiguës de son réseau; l'observation est valable pour l'exploitant de deux lignes partageant un même terminus comme pour les principales sociétés, aux réseaux départementaux interconnectés. Ces pratiques supposaient l'absence d'obstacles techniques : gabarit unifié et, pour les engins de traction, adaptation au profil... 
Sur certaines lignes secondaires à voie normale, le matériel fourni par un réseau d'intérêt général est comptabilisé à ce titre et ne figure pas dans les statistiques propres aux chemins de fer secondaires.

\section{. Les engins de traction}

Les engins de traction comprennent les locomotives et les automotrices définies par l'administration comme des voitures munies d'un moteur quelconque ou empruntant leur force à un conducteur extérieur et renfermant un compartiment pour voyageurs, messageries ou marchandises. Ces automotrices sont en 1911 à vapeur dans la majeure partie des cas; hors les villes, la traction électrique fait une modeste apparition : l'appel au pétrole est évoqué dans un cas ; il se généralisera après 1920. Les exploitants des TVM, dont certains ont l'expérience d'une exploitation urbaine, sont plus enclins que ceux des CFIL à se tourner vers les nouvelles sources d'énergie pour la traction. Les autorails, on le verra, se multiplient comme un moyen de lutter contre la concurrence routière.

\section{$>$ Le parc des CFIL et des TVM}

Le parc des CFIL passe de 605 à 1054 unités dont 17 automotrices, soit $1,6 \%$ du total entre 1901 et 1911 . Ces dernières parcourent quelques lignes à voie étroite, en coexistence avec des locomotives qui tractent certains convois ; ces itinéraires n'ont aucune caractéristique commune sur le triple plan des distances, des profils et du milieu socioéconomiques, qui aurait pu conduire à l'emploi des automotrices (tabl. 41). La traction électrique par fil aérien est employée sur $25 \mathrm{~km}$ au départ de Pierrefitte-Nestalas en direction de Luz-Saint-Sauveur et aussi de Cauterets et La Raillère.

Tableau 41. Nombre d'automotrices en service sur les réseaux des CFIL et lignes concernées

\begin{tabular}{|l|c|c|c|}
\hline \multicolumn{1}{|c|}{ Compagnies } & Km & Locom otives & Autom otrices \\
\hline $\begin{array}{l}\text { Chemin de fer de la vallée de } \\
\text { Celles (Vosges) }\end{array}$ & 24 & 5 & 3 \\
\hline $\begin{array}{l}\text { Chemins de fer } \\
\text { départementaux de l'Aisne }\end{array}$ & 110 & 7 & 3 \\
\hline $\begin{array}{l}\text { Chemins de fer économiques } \\
\text { du Nord }\end{array}$ & 100 & 12 & 4 \\
\hline $\begin{array}{l}\text { Omnibus et tramw ays de } \\
\text { Lyon : Vaugneray à Mornant }\end{array}$ & 4 & 5 & 7 \\
\hline
\end{tabular}


Le parc des TVM passe de 475 engins de traction dont 35 automotrices ( $7 \%$ du total), à 903 engins dont 152 automotrices représentant $17 \%$ du parc en 1911. Les automotrices circulent sur 29 lignes longues de $1147 \mathrm{~km}$; sur 15 d'entre elles, ces véhicules assurent la totalité des circulations, sur les autres, elles partagent ce rôle avec des locomotives. Le premier cas concerne essentiellement de courtes liaisons comprenant un parcours urbain et suburbain développé (Nice, Tours, Grenoble, Cherbourg). Le second cas s'observe dans des réseaux départementaux sur lesquels les automotrices en service sont peu nombreuses (Ardèche, Indre, Loiret...). Sur deux lignes circulent des automotrices affectées au seul acheminement des marchandises (tabl. 42). La traction électrique concerne les lignes de la Compagnie genevoise de tramways électriques, de la Compagnie grenobloise de tramways électriques, de la Société de tramways électriques de Tergnier à Saint-Gobain et AnizyPinon et la liaison Viricelles-Chazelles à Saint-Symphorien-surCoise, en tout $115 \mathrm{~km}$. Signalons la survivance de la traction hippomobile employée dans le Tarn entre Laboutarié et Réalmont sur $5 \mathrm{~km}$ (neuf chevaux), également près de Poitiers, par la Société des tramways de la Vienne (quatre chevaux), enfin dans l'Isère par la Société des chemins de fer économiques du Nord (neuf chevaux à Vienne).

Les principales compagnies bien pourvues en locomotives (62 pour la Compagnie des chemins de fer vicinaux, 52 pour la Compagnie des chemins de fer départementaux) n'utilisaient pas d'automotrice en $1911^{10}$. Les réseaux départementaux disposaient d'un nombre d'engins de traction variant avec le kilométrage exploité et le trafic à assurer : 39 locomotives sur le réseau des Ardennes, 36 pour l'Aude, seulement 11 plus deux automotrices en Ardèche. Les sociétés ou particuliers exploitant une ou deux lignes de quelques dizaines de kilomètres, parfois isolées l'une de l'autre, employaient deux à quatre engins de traction et faisaient souvent appel à des automotrices. A titre d'exemple, quatre locomotives roulent sur la ligne de Pontarlier à Mouthe $(30 \mathrm{~km})$, deux sur les lignes de Taulignan à Grignan et de Tarascon-surAriège à Auzat (11 et $16 \mathrm{~km}$ ); le parc le plus fréquent pour ce type de ligne est constitué de trois locomotives.

10- Le matériel utilisé par la SGCFE a été présenté ci-dessus. 
Tableau 42. Nombre d'automotrices en service en 1911 sur les réseaux des TVM et lignes concernées

\begin{tabular}{|c|c|c|c|}
\hline Compagnies, lignes ou réseaux & Km & Locomotives & Autom otrices \\
\hline \multicolumn{4}{|l|}{ Chemins de fer du sud de la France: } \\
\hline Levens à St-Martin-Vésubie & 34 & 0 & 4 \\
\hline Cagnes à Grasse et Vence & 36 & 0 & 7 \\
\hline La Tour-du-Pin à Avenières & 18 & 2 & 2 \\
\hline \multicolumn{4}{|l|}{ Chem in de fer du département de l'Aisne: } \\
\hline Laon à Nouvion-le-Vieux & 14 & 2 & 3 \\
\hline Réseau de TVM de Saumur : Saumur et banlieue & 21 & 4 & 3 \\
\hline Chem in de fer d'Arpajon : Paris-les-Halles à Arpajon & 36 & 22 & 14 \\
\hline \multicolumn{4}{|l|}{ Compagnie de tramways de Nice et du littoral : } \\
\hline Nice à Contes & 14 & 8 & 6 \\
\hline Nice à Levens & 19 & 0 & 3 \\
\hline Contes à Bendéjun & 3 & 0 & 1 \\
\hline Cagnes à Cap-d'Antibes & 14 & 0 & 3 \\
\hline Port-de-Peille à Grave-de-Peille & 7 & 0 & 1 \\
\hline Compagnie genevoise de tramways électriques & 16 & 3 & 10 \\
\hline $\begin{array}{l}\text { Réseau de T.V.M. de Cherbourg : Cherbourg et } \\
\text { banlieue }\end{array}$ & 11 & 5 & 12 \\
\hline Nord : Cassel-gare à Cassel-ville & 4 & 0 & $3(\mathrm{a})$ \\
\hline Ille-et-Vilaine : Dinard à Saint-Briarc & 9 & 4 & 1 \\
\hline $\begin{array}{l}\text { Rhône et Loire : Viricelles-Chazelles à Saint- } \\
\text { Symphorien-sur-Coise }\end{array}$ & 10 & 2 & 3 \\
\hline Aveyron : Rodez-gare à Rodez-Palais-de-justice & 2 & 0 & 5 \\
\hline $\begin{array}{l}\text { Société de tramw ay électrique de Tergnier à Saint- } \\
\text { Gobain et Anizy-le-Pinon }\end{array}$ & 31 & 1 & $3(b)$ \\
\hline $\begin{array}{l}\text { Compagnie des TVM de Tours : Tours à Saint- } \\
\text { Avertin }\end{array}$ & 5 & 0 & 4 \\
\hline Territoire de Belfort : Dasle à Beaucourt & 4 & 0 & 2 \\
\hline Gironde : Bordeaux à Beychac et Cailleau & 12 & 0 & 4 \\
\hline \multicolumn{4}{|l|}{ Société grenobloise de tramway électrique : } \\
\hline Grenoble à Veurey & 15 & 0 & 3 \\
\hline Grenoble à Chapareillan & 43 & 0 & 11 \\
\hline Chemins de fer départementaux de la Drôme & 195 & 27 & 3 \\
\hline Société des voies ferrées du Dauphiné & 66 & 7 & 11 \\
\hline Réseau des tramways de l'Indre & 182 & 16 & 3 \\
\hline Compagnie de tramways du Loiret & 135 & 12 & 1 \\
\hline Compagnie des tramways de l'Ardèche & 141 & 13 & 2 \\
\hline $\begin{array}{l}\text { Chem ins de fer départementaux de la Haute- } \\
\text { Vienne }\end{array}$ & 50 & 0 & 21 \\
\hline
\end{tabular}




\section{$>$ Le poids des locomotives}

Les documents ministériels fournissent les poids bruts à vide minimum et maximum des locomotives parcourant chaque ligne ou chaque réseau. Le poids minimum, facteur d'adhérence, commande en partie la capacité de traction d'une machine, son aptitude à entraîner, en rampe, sans patiner un convoi d'un tonnage déterminé. Le poids maximum suppose des exigences en matière de résistance des ouvrages d'art et de la voie, de poids des rails; il a des incidences sur les coûts de construction. Les poids bruts des locomotives circulant sur voie étroite se situent dans une fourchette comprise sauf exception entre 15 et $25 \mathrm{t}$ avec des niveaux moyens de 20,4 et 16,9 t pour les CFIL, de 18,8 et 15,6 $\mathrm{t}$ pour les TVM. Ces moyennes sont stables dans la première décennie du $\mathrm{Xx}^{\mathrm{e}}$ siècle. Certaines compagnies font appel à des matériels légers : autour de $10 \mathrm{t}$ pour les Tramways de la Sarthe ou la Compagnie meusienne, 7,6 t entre La Trinité et Etel dans le Morbihan. Quelques compagnies utilisent des locomotives de plus de $25 \mathrm{t}$ sur voie métrique : réseaux du Cher et de la Nièvre de la SGCFE (27,2 et 26,8 t), ligne de Toulon à Fréjus des Chemins de fer du sud de la France (31,3 t), Chemins de fer de la banlieue de Reims $(32,2 \mathrm{t})$.

La Compagnie des chemins de fer départementaux cherche constamment à améliorer ses moyens de traction; elle a le souci de l'harmonisation entre le poids des engins et la qualité de la voie. Sa première ligne, en service en 1885 entre Château-Renault et Port-Boulet, est dotée de rails de $15 \mathrm{~kg} /$ mètre limitant à $5,5 \mathrm{t}$ par essieu le poids des engins de traction; pour les lignes ultérieures les données sont de $18 \mathrm{~kg} / \mathrm{m}$ et $8 \mathrm{t}$. Des machines Compound sont mises en service pour accroitre la vitesse sur les lignes du Vivarais ; on y utilise en 1925 une locomotive semi-Diesel livrée par Thomson-Houston pour faire des économies de traction. La politique de la compagnie est marquée d'une double fidélité, d'abord à des constructeurs, une firme liégeoise, administrateurs obligent, également Fives-Lille et la SACM de Belfort, ensuite a quelques modèles mis en service sur de longues périodes, 40 à 60 ans $^{11}$.

Le poids des automotrices a enregistré une forte augmentation en dix ans s'élevant de 7,5 à 13,6 t; cette évolution traduit les progrès réalisés dans leur construction et dans leur capacité de transport; elle n'empêche pas la circulation, encore en 1911, de matériel très léger, $(4,4$ t) sur les Chemins de fer de la Drôme.

11- J.-C. Riffaud, "Le matériel de traction du CFD», Magazine des Tramways à vapeur et des secondaires, $\mathrm{n}^{\circ} 48$ (1988-4), 54 p., et $\mathrm{n}^{\circ} 49$ (1989-1), $57 \mathrm{p}$. 
Les poids bruts des locomotives circulant sur voie normale se situent dans la fourchette comprise entre 20 et $30 \mathrm{t}$ avec quelques exceptions, l'écart est de $40 \mathrm{t}$ entre les 47,2 t de la machine parcourant la ligne Senones à Étival et les $7 \mathrm{t}$ de la locomotive de la Société des chemins de fer économiques et forestiers des Landes. Le profil des itinéraires assurés par les machines devait peser sur le choix du matériel de traction, mais la nature du trafic était sans doute un facteur plus décisif. Une saine gestion exigeait d'adapter le matériel à la demande pour éviter un surplus de consommation d'énergie, aussi des locomotives de différents types se trouvaient-elles sur de mêmes liaisons. La Compagnie des chemins de fer départementaux des Bouches-du-Rhône utilisait des locomotives de $30 \mathrm{t}$ pour acheminer ses trains «lourds » chargés des produits des carrières de Fontvieille, mais aussi des machines de $18 \mathrm{t}$ pour des services de voyageurs et de messageries. Le parc des engins de traction des chemins de fer secondaires comporte enfin un matériel adapté à l'exploitation des lignes à crémaillère et des funiculaires ${ }^{12}$.

\section{. Les voitures et fourgons}

Le parc des chemins de fer secondaires s'enrichit de 2185 voitures et 577 fourgons entre 1901 et 1911 ; il est alors de 4941 voitures et 1176 fourgons. Les automotrices déjà inventoriées parmi les engins de traction sont à nouveau comptabilisées avec les voitures, puisqu'elles répondent à une double fonction. Ces matériels forment les trains de voyageurs et, associés à des wagons, les trains voyageurs-marchandises. Les fourgons transportent les messageries et certaines expéditions en « Grande Vitesse » confiées aux trains de voyageurs. Les compagnies exploitant de courtes lignes disposent de deux ou de trois fourgons.

Le parc comprenait des voitures de $1^{\mathrm{re}}, 2^{\mathrm{e}}, 3^{\mathrm{e}}$ classes, des voitures à classe unique, des voitures mixtes associant deux classes ou comportant, à côté d'un compartiment pour les voyageurs, un espace aménagé pour les bagages et la poste, disposition évitant la circulation d'un fourgon. Les compagnies poursuivaient deux objectifs contradictoires : rechercher des économies de traction et d'entretien des matériels en allégeant la composition des trains, offrir divers niveaux de confort adaptés aux exigences de clientèles variées. Le confort changeait avec les classes, le nombre de places par voiture en était l'expression ${ }^{13}$. Les

12- Voir comment la création littéraire rend compte des économies faites par les compagnies sur le matériel dans «Le Tacot», nouvelle de Jean-Richard Bloch parue en 1912 dans le recueil Léry. Premier livre de contes (Paris, NRF).

13- Ces questions techniques sont minutieusement décrites dans l'Agenda Dunod, ouvr. cité, cf. p. 152-158. 
voitures mixtes et celles de $2^{\mathrm{e}}$ et $3^{\mathrm{e}}$ classe offraient entre 43 et 51 places sur les lignes à voie normale, entre 31 et 38 sur les lignes à voie étroite ; le nombre était de 21 ou 22 dans les deux cas, en $1^{\text {re }}$ classe. Les voitures à classe unique, absentes sur les lignes à voie normale, avaient entre 29 et 43 places $^{14}$. Les voitures étaient évidemment plus lourdes sur voie normale (6 à 10 t) que sur voie étroite (4 à 7,9 t), les TVM employant le matériel le plus léger. Les automotrices disposaient d'aménagements propres à répondre à la demande sur les lignes desservies : souvent deux classes pour les voyageurs et un compartiment pour les bagages et messageries ; tous les cas de figures se retrouvaient.

La place disponible par voyageur, le chauffage et l'éclairage sont trois facteurs essentiels pour le confort des voyageurs. Les améliorations, lentes à être adoptées, varient suivant les compagnies. Le chauffage avec deux bouillottes par compartiment remplies avant le départ par divers procédés, l'éclairage à la bougie ou à la lampe à pétrole donné par un lumignon sont d'usage courant au début du siècle. Les progrès décisifs viennent avec l'éclairage électrique et le chauffage à vapeur.

Le parc évolue en dix ans vers un matériel plus lourd, résistant et stable : le poids moyen des voitures de $3^{\mathrm{e}}$ classe passe de $3,5 \mathrm{t}$ à $4,5 \mathrm{t}$ (tabl. 43).

Le parc évolue aussi vers la simplification de l'offre. Les CFIL ont conservé un temps le régime des trois classes hérité de leur origine et imité des grandes compagnies, notamment sur leurs lignes à voie normale. Les TVM naissent avec deux classes. Les matériels permettant de réduire le nombre de voitures d'un train, donc la charge remorquée et la consommation d'énergie, se multiplient: automotrices, voitures mixtes, voitures de $2^{\mathrm{e}}$ classe pouvant être associées aussi bien à des voitures de $1^{\text {re }}$ que de $3^{\text {e }}$ classe (tabl. 44).

L'offre limitée à deux classes se partageait inégalement entre une classe inférieure avec le « confort » de la $3^{\mathrm{c}}$ classe, même définie comme $2^{\mathrm{e}}$ classe, et une classe supérieure appelée $1^{\text {re }}$ classe où les voyageurs retrouvaient les voitures de $2^{\mathrm{e}}$ classe préférées par les compagnies à celles de $1^{\text {re }}$, leur capacité étant d'environ $50 \%$ supérieure. La circulation des véritables voitures de $1^{\text {te }}$ classe était limitée, la tarification semblait établie en fonction des caractéristiques des voitures et non de la désignation des classes.

14- Les caractéristiques propres au matériel en service sur les lignes de la SGCFE figurent au chap. Iv, p. 133-137 et p. 151-152. 


\begin{tabular}{|c|c|c|c|c|c|c|c|}
\hline \multirow{5}{*}{$\sum$} & 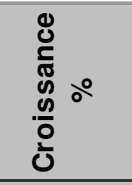 & $\bar{\sim}$ & R & $\stackrel{\llcorner}{\infty}$ & $\begin{array}{l}\underset{1}{\sim} \\
\stackrel{1}{1}\end{array}$ & ๑ூ & 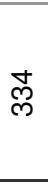 \\
\hline & 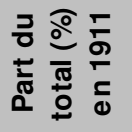 & $\begin{array}{l}\infty \\
0^{-}\end{array}$ & $\begin{array}{l}\dot{J} \\
\dot{L}\end{array}$ & $\stackrel{\sim}{\sim}$ & $\begin{array}{l}0 \\
\stackrel{\sim}{N}\end{array}$ & $\stackrel{\stackrel{N}{\sim}}{\stackrel{N}{N}}$ & $\stackrel{6}{6}$ \\
\hline & 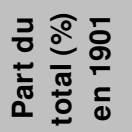 & $\hat{\sigma}$ & 员 & $\nabla$ & $\underset{0}{\stackrel{N}{0}}$ & $\stackrel{\text { N }}{\text { N }}$ & 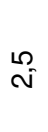 \\
\hline & 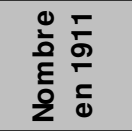 & $\underset{\widetilde{\sigma}}{ }$ & $\begin{array}{l}\stackrel{\mathscr{D}}{N} \\
\stackrel{-}{-}\end{array}$ & $\stackrel{\text { ષั }}{ }$ & $\mathscr{6}$ & ชి & ָָ \\
\hline & 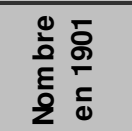 & 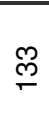 & Ñ & $\stackrel{6}{\circ}$ & $œ$ & ஜొ & ભొ \\
\hline \multirow{5}{*}{ 壱 } & 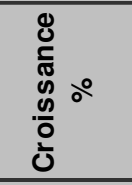 & $\stackrel{\infty}{\bullet}$ & $\stackrel{m}{\longrightarrow}$ & ले & $\varliminf_{1}$ & $\bar{\infty}$ & \\
\hline & 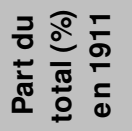 & $\stackrel{\infty}{\sim}$ & $\frac{1}{\sigma}$ & $\begin{array}{l}\mathscr{L}^{\circ} \\
\stackrel{6}{6}\end{array}$ & $\overline{0}$ & $\begin{array}{l}\infty \\
\infty^{\infty} \\
\infty\end{array}$ & $\stackrel{m}{O}$ \\
\hline & 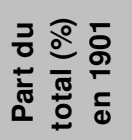 & $\stackrel{\sim}{\sim}$ & $\bar{m}$ & $\stackrel{\text { N }}{\mathcal{N}}$ & $\stackrel{\sigma}{\circ}$ & 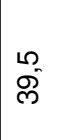 & \\
\hline & 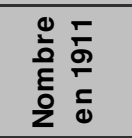 & $\stackrel{N}{N}$ & 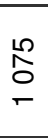 & 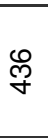 & $\nabla$ & পু & $\stackrel{\wedge}{\sim}$ \\
\hline & 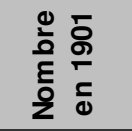 & 寸 & $\underset{+}{\mathscr{q}}$ & $\underset{\sim}{\mathbb{N}}$ & $\stackrel{\sim}{\simeq}$ & 온 & \\
\hline & & 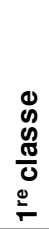 & $\begin{array}{l}0 \\
0 \\
\stackrel{2}{0} \\
\frac{\pi}{0} \\
\stackrel{0}{N}\end{array}$ & 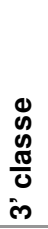 & $\begin{array}{l}0 \\
\frac{0}{5} \\
\frac{0}{5} \\
5 \\
0 \\
0 \\
\frac{0}{0} \\
\frac{\pi}{0}\end{array}$ & 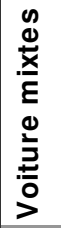 & 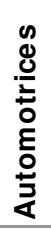 \\
\hline
\end{tabular}


Les trains circulant sur des dizaines de kilomètres à travers des zones rurales génératrices de faible trafic présentaient plusieurs types de composition : les nombreux convois marchandises-voyageurs comprenaient une ou deux voitures de classe inférieure et une voiture mixte offrant des places en classe supérieure et un espace pour les bagages ; cette disposition a été adoptée par les grandes compagnies sur les lignes peu fréquentées, lorsque l'obligation d'offrir trois classes a été levée. Des voitures supplémentaires étaient prévues les jours de marchés et surtout de pèlerinages ou de foires dans la mesure où les conditions de traction et d'exploitation le permettaient.

La persistance de deux classes sur les chemins de fer secondaires ne saurait surprendre lorsqu'on constate leur maintien dans les années trente dans les autobus parisiens et dans le métro jusqu'en 1986.

Chaque exploitant se dote d'un parc de voitures ; quels ont été les critères de choix dans la politique d'achat du matériel ? L'inventaire des voitures utilisées sur les divers réseaux des plus grandes entreprises montre une diversité qui suggère la recherche d'une adaptation à la demande.

La SGCFE privilégie les voitures mixtes partout utilisées. L'ensemble formé par les réseaux voisins et contigus de l'Allier, du Cher et de la Nièvre, $42 \%$ des kilomètres exploités par la compagnie, n’utilise que $30 \%$ des voitures, l'utilisation du matériel étant favorable. À l'opposé, sur une ligne isolée de $16 \mathrm{~km}$, représentant $1,9 \%$ du réseau, circulent 12 voitures, soit 3,5\% du parc. La Compagnie des chemins de fer vicinaux a un réseau de CFIL à voie étroite et un réseau de TVM, chacun doté de son propre matériel ; le premier offre trois classes, le second deux. Les voitures mixtes sont peu utilisées, $7 \%$ du parc contre $58 \%$ pour la SGCFE, $39 \%$ pour la Compagnie des chemins de fer départementaux. Cette dernière utilise un total de 148 voitures sur ces six réseaux ; seul le réseau de Seine-et-Marne offre trois types de voitures $\left(2^{\mathrm{e}}, 3^{\mathrm{e}}\right.$ classe et mixtes) les autres (Charente, Indre-et-Loire, Manche, Saône-et-Loire, Yonne) seulement deux ( $3^{\mathrm{e}}$ classe et mixte).

Les compagnies moins importantes exploitant un réseau dans un cadre départemental utilisent toutes des voitures de $2^{\mathrm{e}}$ classe et des voitures mixtes dont l'aménagement fait voisiner le compartiment à bagages avec des compartiments pour voyageurs de $3^{\text {e }}$ classe, plus rarement de $1^{\text {te }}$ classe (Ardèche, Ardennes). Les voitures de $1^{\text {te }}$ classe sont rares : une dans la Sarthe ou l'Aude, sans doute utilisée pour des circulations spéciales. La Compagnie des tramways de l'Indre ne dispose que de 22 voitures mixtes et trois automotrices. Les exploitants de lignes isolées ont un parc de l'ordre de deux ou trois à dix voitures en fonction du kilométrage et de la nature de la desserte : trois voitures 
mixtes entre Cassel-gare et Cassel-ville, deux voitures mixtes et cinq voitures de $2^{\mathrm{e}}$ classe entre Challans et Fromentine. À ce niveau d'équipement, l'immobilisation d'un véhicule conduisait à l'irrégularité du service.

\section{. Les wagons}

Les wagons circulant sur les réseaux secondaires étaient au nombre de 10014 en 1901, de 18897 en 1911, soit une progression de $88 \%$; ce parc appartenait aux CFIL pour $63 \%$, aux TVM pour $37 \%$. La capacité totale du parc des deux réseaux atteignait en 1911115155 t, les CFIL en détenant les deux tiers. La capacité moyenne des wagons n'a pas changé dans la première décennie du siècle, elle était respectivement de 9,5 $\mathrm{t}$ pour les CFIL et $8,5 \mathrm{t}$ pour les TVM.

Les statistiques ne donnent ni les types de wagons, ni le nombre de véhicules de service qui est inclus (ballast, combustible, secours). Les monographies sur les sociétés dressent des inventaires permettant de pallier cette lacune. Les réseaux utilisaient des wagons couverts, des wagons tombereaux et des wagons plates-formes; certaines lignes connaissaient le développement particulier d'un trafic : bétail, céréales, matériaux de construction, minerais... ; le parc devait s'adapter à la demande en privilégiant un type de wagon ; contrairement aux réseaux d'intérêt général dont l'extension conduisait à la diversité des trafics, les réseaux secondaires pouvaient limiter la diversité de leur parc.

Plus du quart du matériel en service sur les réseaux secondaires dessert les lignes de trois sociétés : SGCFE $16 \%$, chemins de fer vicinaux 5,3\%, Chemins de fer départementaux 5,1\%. Comme pour les voitures, un réseau étendu autorise une bonne rotation du matériel : on retrouve, pour la SGCFE, $42 \%$ du kilométrage exploité avec $34 \%$ du parc de wagons sur les lignes de l'Allier, du Cher et de la Nièvre. L'intensité du trafic est un autre facteur favorable à une bonne utilisation du matériel : le rapport du nombre de wagons aux kilomètres desservis est de 1,3 pour les trois départements cités ; sur le réseau de la Somme de la même compagnie il s'élève à 2,4. L'écart est encore plus sensible entre les six réseaux de la Compagnie des chemins de fer départementaux : 0,7 en Indre-et-Loire, 2,6 en Seine-et-Marne, où le trafic des betteraves était volumineux.

La comparaison du même rapport pour des compagnies plus modestes conduit à des résultats de même ordre pour les CFIL (Réseau du Morbihan 0,3, Réseau Est de Lyon 4,4) comme pour les TVM (Chemins de fer et tramways du Midi de la France 0,1, Chemins de fer de Voiron à Saint-Béron 3,9). Le nombre de wagons en valeur absolue est faible sur les lignes courtes: trois wagons et une automotrice 
marchandises entre Cassel-gare et Cassel-ville, 23 wagons entre SaintVictor et Thizy, 32 wagons entre Pontarlier et Mouthe. Pour certaines compagnies, les données ne permettent pas d'approcher la réalité. Sur les lignes à voie normale, le matériel est souvent fourni par les grandes compagnies, notamment l'Est. Le prêt de matériel entre compagnies dont les réseaux sont compatibles trouble la rigueur statistique : la Compagnie des chemins de fer du sud de la France emprunte du matériel à la Compagnie des tramways de Nice et du littoral, notamment entre Saint-Tropez et Cogolin ; la Compagnie des tramways de Cherbourg achemine ses expéditions en petite vitesse dans les wagons de la Société des carrières de l'Ouest. Ces quelques exemples, entre d'autres, incitent à la prudence.

\section{L’inégale répartition de la dotation en matériel de transport}

Les voitures, fourgons, wagons et automotrices peuvent être comptés chacun pour une unité. Le rapport entre ce parc total et le kilométrage auquel il est affecté $(\mathrm{p}: \mathrm{km})$ exprime un niveau d'équipement, une plus ou moins grande capacité de transport. Les statistiques fournissent ces données tantôt pour une entreprise, tantôt pour un réseau, tantôt pour une ligne. Le coefficient moyen est de 1,64 pour l'ensemble des réseaux de CFIL et de 1,43 pour celui des TVM. L'analyse portant sur 204 liaisons en révèle $14(6,5 \%)$ particulièrement pauvres en matériel avec un coefficient inférieur à 0,9 et $23(11,2 \%)$ très bien dotées avec un coefficient dépassant 3. Cent trois lignes $(50,9 \% \mathrm{du}$ total) offrent un coefficient compris entre 1,1 et 2 .

Les liaisons bien équipées sont, à l'exception de trois d'entre elles, des lignes de moins de $40 \mathrm{~km}$, souvent même de $10 \mathrm{~km}$. Huit relèvent des réseaux à voie normale des CFIL qui ont conservé du matériel de leur origine. Deux coefficients très élevés correspondent à des situations particulières : la Société Malétra $(\mathrm{P}: \mathrm{km}=20)$ exploite deux kilomètres entre Rouen et Petit-Quevilly avec 40 wagons pour des trafics portuaires et industriels ; la Société nationale des chemins de fer vicinaux de Belgique $(\mathrm{P}: \mathrm{km}=87)$ dispose de deux kilomètres en France entre la frontière et Steenverck, prolongement d'une ligne belge desservie par un parc de 174 unités. Deux ensembles de départements abritent ces relations privilégiées : un groupe très important incluant l'Aisne, la Somme, le Pas-de-Calais et surtout le Nord, un autre autour du Rhône avec la Loire, l'Isère et la Savoie. Ces départements ont en commun l'existence d'une activité industrielle. Les autres départements concernés sont dispersés géographiquement, quelques-uns comptent une grande ville (Paris, Bordeaux, Nice) (tabl. 44). 
Tableau 44. Réseaux ou lignes bien dotés en matériel

\begin{tabular}{|c|c|c|c|c|c|c|}
\hline Ré gime & Écartement & Compagnie, ligne ou réseau & Départements & $\mathrm{Km}$ & Parc & $\mathrm{P} / \mathbf{k m}$ \\
\hline CFIL 1865 & Normal & $\begin{array}{l}\text { Société Malétra : Rouen à Petit- } \\
\text { Quevilly }\end{array}$ & Seine-Maritime & 2 & 40 & 20 \\
\hline CFIL 1865 & Normal & Réseau de l'est de Lyon & \begin{tabular}{|l|}
$\begin{array}{l}\text { Rhône, } \\
\text { Isère }\end{array}$ \\
\end{tabular} & 105 & 551 & 5,2 \\
\hline CFIL 1865 & Normal & $\begin{array}{l}\text { Compagnie de Gué à } \\
\text { Ménaucourt et embranchement }\end{array}$ & \begin{tabular}{|l} 
Meuse, \\
Haute-Marne
\end{tabular} & 36 & 162 & 4,5 \\
\hline CFIL 1865 & Normal & $\begin{array}{l}\text { Compagnie du chemin de fer de } \\
\text { Saint-Quentin à Guise }\end{array}$ & Aisne & 48 & 199 & 4,1 \\
\hline CFIL 1865 & Normal & Vertaizon à Billom & Puy-de-Dôme & 9 & 29 & 3,2 \\
\hline CFIL 1880 & Normal & $\begin{array}{l}\text { Compagnie du Médoc : Pauillac à } \\
\text { Port-des-pilotes }\end{array}$ & Gironde & 1 & 4 & 4 \\
\hline CFIL 1880 & Normal & Marquion à Cambrai & $\begin{array}{l}\text { Nord, } \\
\text { Pas-de-Calais }\end{array}$ & 20 & 68 & 3,4 \\
\hline CFIL 1880 & Normal & $\begin{array}{l}\text { Compagnie du chemin de fer } \\
\text { d'Aulnoye à Pont-sur-Sambre }\end{array}$ & Nord & 5 & 16 & 3,2 \\
\hline CFIL 1880 & Éroit & $\begin{array}{l}\text { Compagnie CFIL du nord de la } \\
\text { France : Roisel à Hargicourt }\end{array}$ & \begin{tabular}{|l|} 
Aisne, \\
Somme
\end{tabular} & 7 & 49 & 7 \\
\hline CFIL 1880 & Éroit & SGCFE : groupe du nord & Nord & 10 & 54 & 5,4 \\
\hline TVM & & $\begin{array}{l}\text { Société nationale des chemins } \\
\text { de fer vicinaux belges: } \\
\text { Steenverck-frontière }\end{array}$ & Nord & 2 & 174 & 87 \\
\hline TVM & & \begin{tabular}{|l|} 
Compagnie des tramw ays de \\
Nice et du littoral : Nice à Contes
\end{tabular} & Alpes-Maritimes & 14 & 92 & 6,5 \\
\hline TVM & & Pithiviers à Toury & \begin{tabular}{|l} 
Loiret, \\
Eure-et-Loir
\end{tabular} & 31 & 186 & 6 \\
\hline TVM & & Paris-les-halles à Arpajon & $\begin{array}{l}\text { Seine, } \\
\text { Seine-et-Oise }\end{array}$ & 36 & 208 & 5,7 \\
\hline TVM & & Rodez : Gare-palais de justice & Aveyron & 2 & 10 & 5 \\
\hline TVM & & Ardres à Pont-d'Ardres & Nord & 6 & 27 & 4,5 \\
\hline TVM & & $\begin{array}{l}\text { Voiron à Saint-Béron et à } \\
\text { Fourvoirie }\end{array}$ & $\begin{array}{l}\text { Isère, } \\
\text { Savoie }\end{array}$ & 36 & 159 & 4,4 \\
\hline TVM & & $\begin{array}{l}\text { Société du tramw ay de la vallée } \\
\text { d'Hérimoncourt : Audincourt à } \\
\text { Montbéliard et à Hérimoncourt et } \\
\text { Valentigney } \\
\end{array}$ & Doubs & 18 & 77 & 4,2 \\
\hline TVM & & Saint-Victor - Thizy & $\begin{array}{l}\text { Loire, } \\
\text { Rhône }\end{array}$ & 7 & 29 & 4,1 \\
\hline TVM & & $\begin{array}{l}\text { Pontcharra PLM à La Rochette et } \\
\text { à Allevard }\end{array}$ & \begin{tabular}{|l} 
Isère, \\
Savoie
\end{tabular} & 17 & 65 & 3,3 \\
\hline TVM & & Bordeaux à Cadillac & Gironde & 32 & 114 & 3,6 \\
\hline TVM & & Beaucourt à Dasle & $\begin{array}{l}\text { Territoire de } \\
\text { Belfort }\end{array}$ & 4 & 14 & 3,5 \\
\hline TVM & & $\begin{array}{l}\text { Société des voies ferrées du } \\
\text { Dauphiné }\end{array}$ & Isère & 66 & 228 & 3,4 \\
\hline
\end{tabular}


Tableau 45. Réseaux et lignes faiblement dotés en matériel

\begin{tabular}{|c|c|c|c|c|c|c|}
\hline Régime & Écartement & $\begin{array}{c}\text { Compagnie, lignes, } \\
\text { réseaux }\end{array}$ & Départements & Km & Parc & $\mathrm{P} / \mathrm{km}$ \\
\hline CFIL 1865 & Normal & $\begin{array}{l}\text { Compagnie du Médoc: } \\
\text { Margaux à Castelmau }\end{array}$ & Gironde & 9 & 5 & 0,55 \\
\hline CFIL 1880 & Normal & Luxey à Mont-de-Marsan & Landes & 45 & 25 & 0,55 \\
\hline CFIL 1880 & Normal & $\begin{array}{l}\text { Société des chemins de } \\
\text { fer du Born et du } \\
\text { Marensin }\end{array}$ & Landes & 117 & 4 & 0,03 \\
\hline CFIL 1880 & Normal & $\begin{array}{l}\text { CFIL du département des } \\
\text { Landes }\end{array}$ & Landes & 185 & 21 & 0,11 \\
\hline CFIL 1880 & Étroit & $\begin{array}{l}\text { Olonzac à Félines } \\
\text { Hautpoul }\end{array}$ & Aude, Hérault & 19 & 16 & 0,84 \\
\hline CFIL 1880 & Étroit & $\begin{array}{l}\text { Compagnie des tramw ays } \\
\text { de Cherbourg: } \\
\text { Querqueville à Urville }\end{array}$ & Manche & 4 & 3 & 0,75 \\
\hline TVM & & $\begin{array}{l}\text { Compagnie des tramw ays } \\
\text { de Nice et du littoral : } \\
\text { Cagnes au Cap d'Antibes }\end{array}$ & Alpes-Maritimes & 14 & 11 & 0,78 \\
\hline TVM & & La Trinité-sur-Mer à Etel & Morbihan & 20 & 15 & 0,75 \\
\hline TVM & & $\begin{array}{l}\text { Compagnie du chemin de } \\
\text { fer de Pau à Oloron et } \\
\text { Mauléon }\end{array}$ & $\begin{array}{l}\text { Landes, Gers, } \\
\text { Basses- } \\
\text { Pyrénées }\end{array}$ & 226 & 169 & 0,74 \\
\hline TVM & & $\begin{array}{l}\text { Compagnie des chemins } \\
\text { de fer du sud de la } \\
\text { France : Cogolin à Saint- } \\
\text { Tropez }\end{array}$ & Var & 9 & 6 & 0,66 \\
\hline TVM & & $\begin{array}{l}\text { Compagnie des } \\
\text { Tramw ays de Nice et du } \\
\text { Littoral : Contes au Pont } \\
\text { de Bendéjun }\end{array}$ & Alpes-Maritimes & 3 & 2 & 0,66 \\
\hline TVM & & $\begin{array}{l}\text { Compagnie des tramw ays } \\
\text { de Nice et du littoral : Nice } \\
\text { à Levens }\end{array}$ & Alpes-Maritimes & 19 & 12 & 0,63 \\
\hline TVM & & $\begin{array}{l}\text { Société des tramw ays de } \\
\text { l'Ardèche }\end{array}$ & Ardèche & 147 & 85 & 0,6 \\
\hline TVM & & $\begin{array}{l}\text { Compagnie des tramw ays } \\
\text { de Nice et du littoral : Pont- } \\
\text { de-Peille au Pont-de-la- } \\
\text { Grave }\end{array}$ & Alpes-Maritimes & 7 & 4 & 0,57 \\
\hline
\end{tabular}


Les liaisons moins favorisées ont entre 4 et $226 \mathrm{~km}$, quatre dépassent $100 \mathrm{~km}$. Les coefficients les plus faibles constatés sur des réseaux landais sont trompeurs, le matériel pour les acheminements en petite vitesse étant fourni par le Midi. Les départements desservis sont divers : on peut souligner le nombre de relations dans les Alpes-Maritimes et le $\operatorname{Var}$ (tabl. 45). Si nous avions relevé le seuil de 0,89 à 0,99 , nous aurions enregistré douze cas supplémentaires de relations faiblement équipées, portant non sur des lignes mais des compagnies exploitant des réseaux départementaux, six de plus de $100 \mathrm{~km}$ dont deux de plus de $300 \mathrm{~km}$; tous ces réseaux sont situés dans des zones spécifiquement rurales (Aude, Côte-d'Or, Mayenne, Morbihan...).

En toute logique, on constate l'adéquation entre le degré d'équipement et les volumes de trafic réalisés en fonction des milieux socio-économiques desservis. Les entreprises principales ne s'y trompaient pas en adaptant leur parc au trafic de leurs divers centres d'exploitation : pour la SGCFE, le coefficient moyen $\mathrm{P} / \mathrm{km}$ était de 1,8 avec des valeurs de 1,1 pour le Cher, 2,7 pour la Somme et 5,6 pour le groupe Nord; pour les Tramways de Nice et du littoral, le coefficient moyen était de 2,1 avec une fourchette comprise entre 6,5 pour la ligne Nice-Contes et 0,5 à 0,8 pour les autres lignes.

L'inventaire du matériel roulant montre la progression du parc au début du $\mathrm{xx}^{\mathrm{e}}$ siècle ; il souligne l'inégale dotation en matériel suivant les types d'entreprises et de lignes. Le matériel d'origine a souvent fait face au trafic pendant toute la durée d'exploitation de certaines compagnies. Le système de freinage a été modernisé par souci de sécurité ; il n'empêche que les gardes-freins encore employés par certaines compagnies témoignent d'archaïsmes dans ce domaine. Ces matériels circulent sur des voies offrant des paramètres de tracé et de profil qui ne conduisent pas à l'amélioration des performances. 


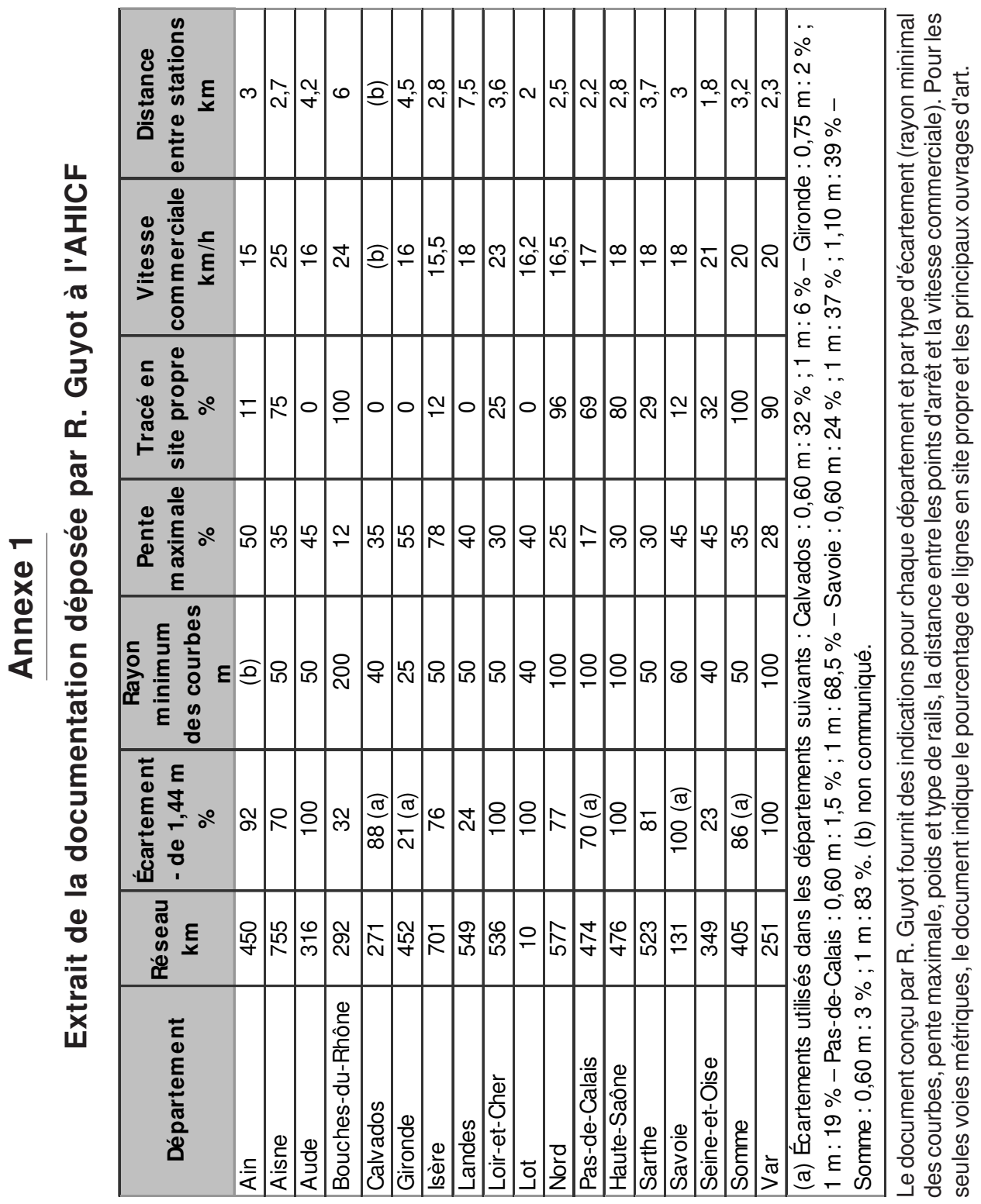




\section{Annexe 2}

\section{Circulaires ministérielles sur le recensement des locomotives (1901 et 1902)}

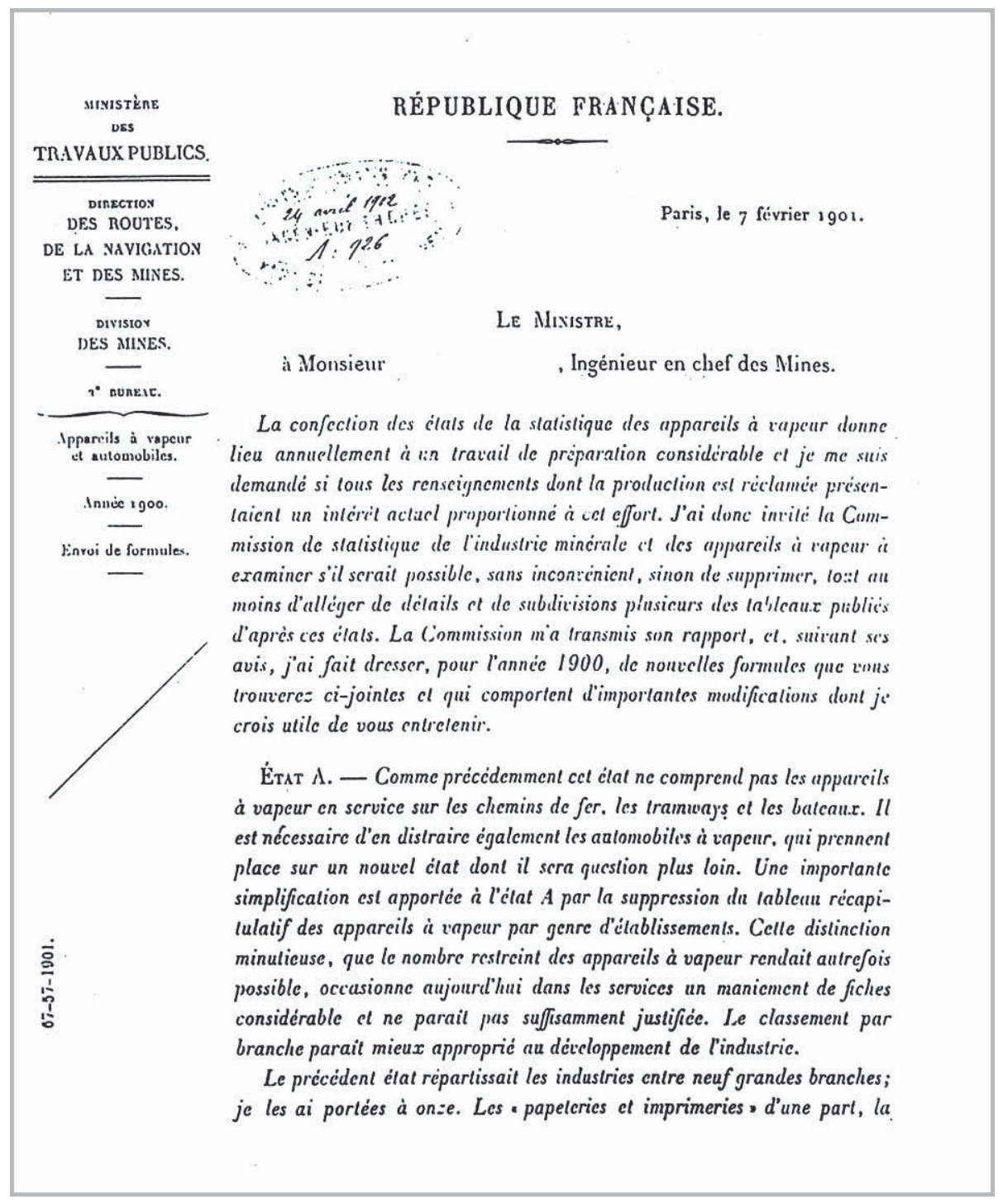




\section{$-2-$}

- production d'énergic électrique , de l'autrc, quifiguraicnt dans des branclies communes à d'autres industries, sonl lobjct dans le préscnt ćlat d'unc rubrique spéciale. La nomenclature des principales industries comprises dans ces onze groupes continue d'aillcurs d'cutrc inscrile en sous-titre sur l'élat, afin de faciliter votre travail de répartition; vous aurcz soin toutefois d'y fairc entrer de vous-méme les industrics qui ny sont pas nominativement désignées.

D'aulre parl, il n'apparafl pas possible de maintenir une division rigoureuse entre les chaudicires molrices el les chaudières simplement calorifères. Il est donc préfcirable de ne pas chicrcher à la fairc el vous n'aurez pluṣ à fournir que le nombre tolal des chaudières en activilic.

De méme, la classificalion des appareils à rapcur d'aprés l'installation des chandicres, qui vous ćlail demandić aussi sur l'ćlat $A$, n'ćlait pas sufisamment définic. Elle est remplacic par une aulre suivant l'espicece, dans laquelle tous les appareils à demcure sont tolalisés, landis que les locomotives pour Icrrassemenls s'y distingucnt des roulcaux compresscurs.

Enfin. Ic tableau des chaudières à rapeur par type est remanie de maniürc à le mellic micux ch rapporl avec les conditions acluclles de lindustric. Il decra comprendre aurcinatant non plus sculement les chandịcres placces à demeure, mais loules les chandieres sans distinction qui fonl l'objcl de l'élal .1.

Ĺtst C. - L'ćlal récapilulalif des locomolives el autres apparcils à vapeur en aclivilé sur les chemins de fer et les tramuay's a ćtć, comme l'élat $A$, profondèment mollifici.

Dans le précédent élal, les renscignemenls èlaienl donnés par entreprise; il y aura lieu, dans lc nouveau, de les fournir par yroupe d'entreprises. Les groupes quifont l'objel d'une rubrique spiciale sont au nombre de cinq :

\section{Réseaux;}

$2^{\circ}$ Autres chemins de fer d'intérèt général.

Les renseignements relatifs à ces deux groupes sont présenlés par les services de contróle et vous n'avez pas à rous en occuper. Il cn est de méme 


\section{$-3-$}

des autres lignes de chemins de fer el de Iramway's placies sous la surveillance de ces services;

3. Lignes d'intérèt local. - Les indicalions lés concernant devront étre tolalisées. Si les chemins de fer s'étendent sur plusicurs départements, vous devrez veiller à ce que les renscignemenls ne soient porlés qu'nne scule fois et vous aurez soin de le faire connaitre par une observation sur l'élal;

$4^{\circ}$ Chemins de fer industriels et divers. - La remarque qui précède s'applique ćyalement à celle calégorie d'entreprises, ainsi qu'à la suivante;

50 Tramways.

Dans chaque groupc les entreprises seront confondues, sans distinction cnire celles qui emploient direclement la vapeur paur la marche des trains el celles qui ne doicent lears mojens de traction qu'au concours de généraleurs fixes, mais vous voudrez bien les désigner nominativement à la premiere paye de lélut.

La longueur kilomélrique des chemins de fer on des tramways est supprimée.

Les types les locomolives sont riduils à deux : les machines $\dot{a}$ foyer et relles sans foyer. Line place est donnée en revanche aux véhicules automobiles à vapeur, qui ne figuraient pas dans le préceilent élat.

L.es apparcils fixes servant à la Iraction sont divisés en quatre calégories, suivant la fonclion qu'ils ont à remplir. Quant aux aulres appareils fixes, on les a réunis sous la niéme rubrique.

Ėта D. - L'étal D relalif aux combustibles consommés par les chemins de fer et les tramuays est le complément de l'élal C. On a donc observé dans sa rédaction le méme groupement par nalure d'entreprises. Toulefois, la dicision par bassin ou pays dorigine a élé mainlenue pour les combuslibles, dans chaque groupe. C"est la seule obscrvation que j'aie à vous faire en ch qui le concerne.

Étır E. - Aucune modificalion n'a ćlć apportéc au lableau des épreuves d'uppureils à rapeur, sauf la suppression du détail des chaudières. Les 


\section{$-4-$}

épreuves de récipients à gaz comprimés ou liquéfiés continucront à élre complées sur ce tableau.

Le tableau des appareils déclarés cst supprimé comme ne présentant pas unc approximation sufisante.

Celui des associations de propriélaircs d'appareils à vapeur n'a subi aucun changement.

Outre les formules $A, C, D, E$ des appareils à vapeur, vous en trouvercz une cinquième, désignée par la lettre $F$ et relative aux aulomobiles autres que ceux des chemins de for et des tramways.

Aux termes des arlicles 7 et 17 du decret du 10 mars 1899 porlant rćglement relalif à la circulation des automobiles, le service des Mines est appelć à constaler, avant la mise en service de ces véhicales, s'ils salisfont aux mesures prescriles par les arlicles 2 à 6. D'autre part, en verlu de larlicle 11, lavis favorable du service des Mines est obligatoire pour la délivrance du cerlificat de capacité cxigé des conducleurs d'automobiles. Le scrvice des Mines est donc à méme de fournir les indications nécessaircs pour permettre de suivre les progrís de celle induslric nouvelle el si inlćressante.

Les intitulés des colonnes du tableau sont suffisamment clairs pour que je n'aic pas besoin d'entrer ici dans des explications superflues. Jc désire que les renscignemenls soient présentés par déparlement el portent, exceptionnellemenl pour celle fois, sur l'cnsemble des opérations failes, d'une part, en 1899, de lautre, en 1900, chacune des deux années devant faire l'objel d'un paragraplic distincl.

Je vous pric de Iransmellire les formules el les exemplaires de la présente circulaire aux Ingénicurs placés sous vos ordres el de leur donner en méme temps les instructions nécessaircs pour que les ćlals me parviennent, remplis, avant le 15 mars prochain.

Par autorisation :

Le Conseiller d'État, Directeur des Routes, de la Navigation et des Mines,

M. JOZON. 


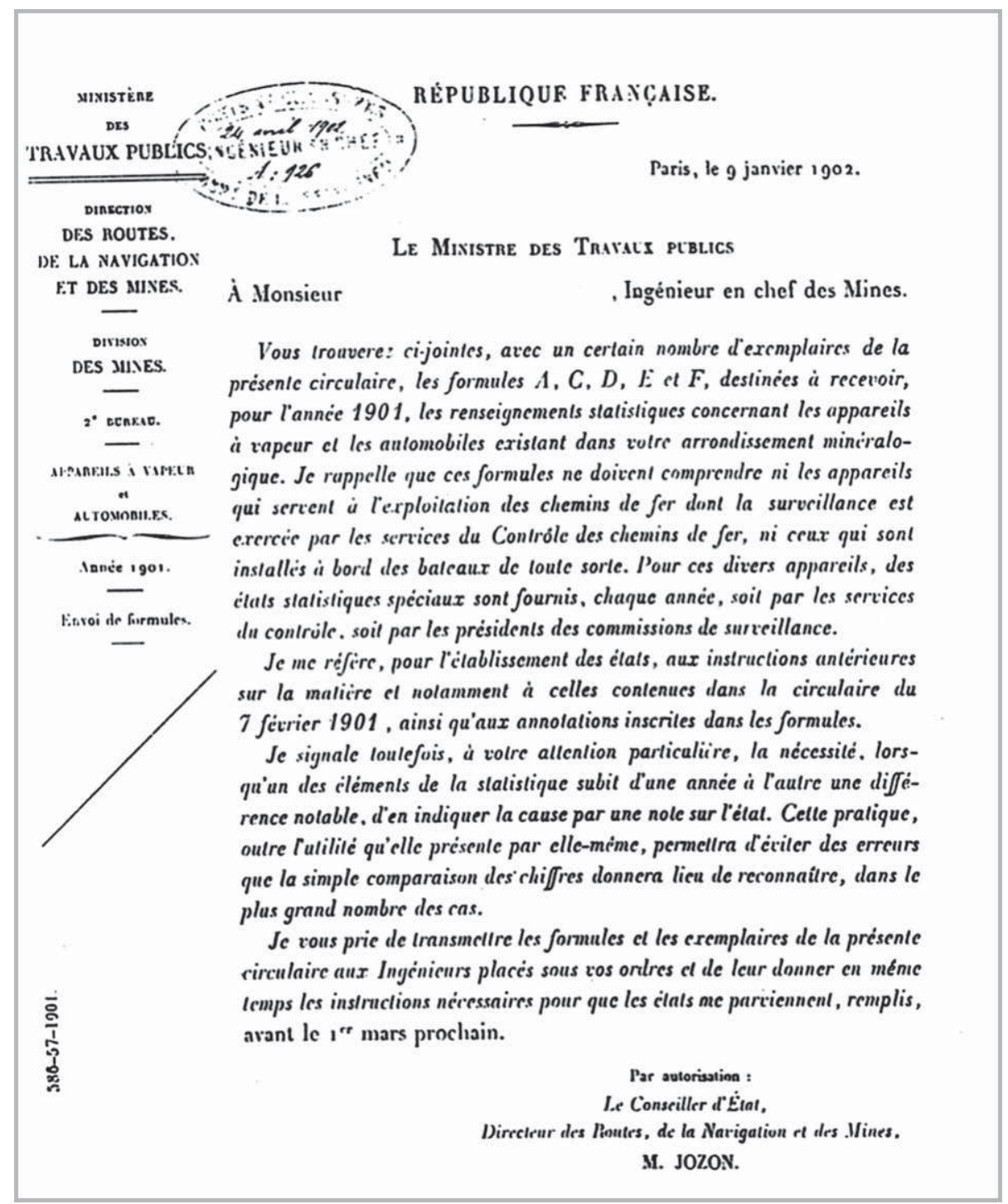

